\title{
Title: Bifunctional small molecules that mediate the degradation of extracellular proteins
}

Authors: David F. Caianiello ${ }^{1}$, Mengwen Zhang ${ }^{1,2}$, Jason D. Ray ${ }^{1}$, Jake C. Swartzel ${ }^{1,3}$, Emily M.

5 J. Branham ${ }^{1}$, Egor Chirkin ${ }^{1}$, Venkata R. Sabbasani ${ }^{1}$, Angela Z. Gong ${ }^{1}$, David M. McDonald ${ }^{1}$, Viswanathan Muthusamy ${ }^{4}$, David A. Spiegel ${ }^{1, *}$

\section{Affiliations:}

${ }^{1}$ Department of Chemistry, Yale University. 225 Prospect Street, New Haven, CT 06511 (USA).

${ }^{2}$ Department of Molecular Biophysics and Biochemistry, Yale University. 266 Whitney Avenue,

New Haven, CT 06511 (USA).

${ }^{3}$ Department of Molecular, Cellular, and Developmental Biology, Yale University. 260 Whitney Ave, New Haven, CT 06511 (USA).

${ }^{4}$ Yale Center for Precision Cancer Modeling, Yale University School of Medicine, New Haven, CT (USA).

*Correspondence to: david.spiegel@yale.edu

Abstract: Targeted protein degradation (TPD) has emerged as a promising and exciting therapeutic strategy. The majority of existing TPD technologies rely on the ubiquitin-proteasome system, and are therefore limited to targeting intracellular proteins. To address this limitation, we developed a class of modularly designed, bifunctional synthetic molecules called MoDE-As (Molecular Degraders of Extracellular proteins through the Asialoglycoprotein receptor (ASGPR)), which are capable of mediating the degradation of extracellular proteins. MoDE-A molecules mediate the formation of a ternary complex between a target protein and the ASGPR, which is expressed primarily on hepatocytes. The target protein is then endocytosed and degraded by lysosomal proteases. We demonstrated the modularity of the MoDE-A technology by synthesizing bifunctional molecules that induce the degradation of both antibody and proinflammatory cytokine proteins. To our knowledge, these data represent the first experimental evidence that non-proteinogenic, synthetic molecules can be employed for the TPD of extracellular proteins both in vitro and in vivo. We believe that TPD mediated by the MoDE-A technology will have widespread applications for disease treatment.

One Sentence Summary: Bifunctional molecules that engage both a target protein and an endocytic receptor are able to induce the lysosomal degradation of extracellular proteins.

\section{Main Text:}

Extracellular protein levels are elevated in many different conditions, and technologies that mediate their removal from circulation have the potential to ameliorate a wide range of disease states $(1,2)$. One mechanism to decrease the serum concentration of an extracellular protein is to induce its endocytosis and subsequent degradation by lysosomal proteases. In order to accomplish 
this goal, we developed a class of bifunctional molecules collectively termed Molecular Degraders of Extracellular proteins through the Asialoglycoprotein receptor (MoDE-As). MoDE-A molecules mediate the formation of a ternary complex between a target protein and the endocytic asialoglycoprotein receptor (ASGPR). The target protein is then endocytosed, trafficked to lysosomes, and degraded by lysosomal proteases (Figure 1A). To validate our technology, we focused on inducing the degradation of a model antibody that binds dinitrophenol (DNP). We also expanded our technology to induce the uptake of the cytokine macrophage migratory inhibitory factor (MIF), which is implicated in numerous inflammatory diseases (3). The bifunctional molecules developed through this work represent the first reported extracellular protein degrading technology validated in vivo.

We chose to degrade proteins via ASGPR-dependent mechanisms for several reasons. ASGPR is primarily expressed on hepatocytes, which are capable of catabolizing large quantities of protein with minimal toxicity compared to other cell types (4-8). Indeed, ASGPR controls the half-lives of several endogenous circulating proteins, including hormones (9), alkaline phosphatases (10), and proteins terminating in Siao2,6Gal glycans (11). ASGPR also plays a role in controlling the concentration of platelets in serum (12). Because of its well-defined binding requirements (13) and its abundant expression on hepatocytes, ASGPR has been used extensively to deliver therapeutic agents to the liver (14-18). Ligands for ASGPR are also readily available. Molecules that display multiple galactose-type sugars - such as the desialylated serum glycoproteins that aided in the discovery of ASGPR (19) - bind strongly to the receptor (20). For example, the ASGPR-binding trivalent GalNAc motif utilized in MoDE-A molecules, which is similar to those used in previous studies (21), is estimated to bind to multimeric ASGPR with $\mathrm{K}_{\mathrm{d}}$ values in the low nanomolar range (22).

ASGPR mediates protein endocytosis through a well-studied mechanism. Each functional receptor 25 is composed of two or more ASGPR protein chains, each with an extracellular C-type lectin domain with modest affinity to N-acetylgalactosamine (GalNAc) and related carbohydrates (23). Following endocytosis, ASGPR's binding to its sugar ligands is disrupted, and the dissociated protein ligand is trafficked to lysosomes where it is ultimately degraded by lysosomal proteases $(24,25)$.

30 The bifunctional MoDE-A molecules developed in this work contain three domains: an ASGPRbinding motif, a PEG spacer, and a protein-binding ligand (Figure 1A). D-MoDE-A and M-MoDEA share the same ASGPR binding motif, but feature different target-binding termini (Figure 1B). To bind to $\alpha$-DNP antibodies, D-MoDE-A contains a dinitrophenyl group connected through a PEG linker to the ASGPR-binding motif. In contrast, the MIF-binding molecule M-MoDE-A was

35 synthesized by incorporating a carboxylic acid-terminated MIF tautomerase inhibitor with a $\mathrm{K}_{\mathrm{d}}$ of approximately $53 \mathrm{nM}(26)$. Full synthetic methods and characterization are available in the supporting information. 


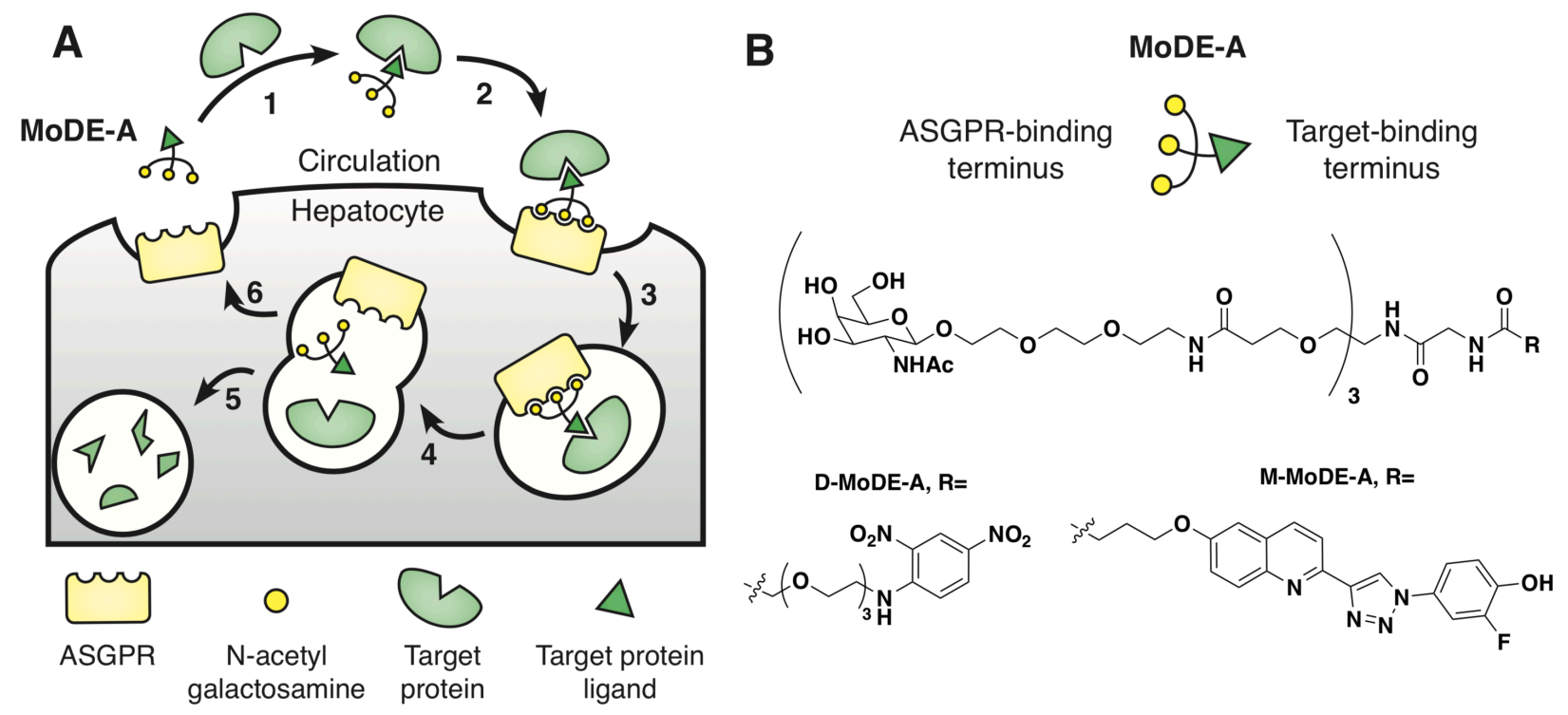

Figure 1: Bifunctional MoDE-A molecule design and chemical structures.

A) Schematic of uptake of target proteins via ASGPR mediated by MoDE-A bifunctional molecules.

B) Chemical structure of bifunctional molecules D-MoDE-A and M-MoDE-A. 
We first sought to investigate whether D-MoDE-A could mediate the formation of a ternary complex between a fluorescently labeled $\alpha$-DNP antibody and ASGPR on the surface of immortalized human hepatocyte HepG2 cells in suspension. The extent of fluorescently-labeled antibody association with cells was found to be dependent on the concentration of D-MoDE-A, with concentrations of $7.4 \mathrm{nM}$ and $0.12 \mu \mathrm{M}$ eliciting half-maximal fluorescence association (Supplemental Figure 2A). The observed bell-shaped response to D-MoDE-A concentration is consistent with the prozone effect commonly observed in systems wherein a ternary complex is formed (27).

10 Cell-associated fluorescence was found to be inhibited by reagents that bind competitively to either ASGPR or $\alpha$-DNP antibody (Supplemental Figure 2B). For example, we synthesized an antibodybinding negative control, DNP-OH3, which contains hydroxyl groups in place of GalNAc residues and is therefore not expected to engage ASGPR (Supplemental Figure 1A). Cellular fluorescence was decreased by increasing concentrations of both DNP-OH3 $\left(\mathrm{IC}_{50}=36 \mathrm{nM}\right)$ and monomeric

15 GalNAc sugar $\left(\mathrm{IC}_{50}=0.20 \mathrm{mM}\right.$ ) (Supplemental Figure 2B). Proteins that bind specifically to ASGPR also decreased D-MoDE-A-mediated cellular fluorescence (Figure 2A). Fetuin (28) and orosomucoid (ORM) (29) are serum glycoproteins that bind to ASGPR only after they have been desialylated to produce asialofetuin $\left(\mathrm{ASF}, \mathrm{K}_{\mathrm{i}}=17 \mathrm{nM}\right)$ and asialoorosomucoid $\left(\mathrm{ASOR}, \mathrm{K}_{\mathrm{i}}=\right.$ $1.7 \mathrm{nM})$, respectively (30). Both ASF $\left(\mathrm{IC}_{50}=65 \mathrm{nM}\right)$ and ASOR $\left(\mathrm{IC}_{50}=17 \mathrm{nM}\right)$ decreased cellular

20 fluorescence, while fetuin and ORM did not. Taken together, these data suggest that D-MoDE-A mediates the formation of a ternary complex between $\alpha$-DNP antibody and ASGPR on the hepatocyte surface.

We next explored whether D-MoDE-A mediates the endocytosis of fluorescently labeled $\alpha$-DNP 25 antibody. We observed that the intracellular fluorescence of adherent HepG2 cells was dependent on the concentration of both D-MoDE-A and antibody (Figure 2B), and that fluorescence increased over time (Supplemental Figure 2C). This observed increase in intracellular fluorescence was inhibitable by reagents that were previously shown to interfere with ternary complex formation (Figure 2C). As expected, we did not observe a significant decrease in antibody uptake when cells 30 were treated with the proteins ORM or fetuin, which do not bind strongly to ASGPR. These data are consistent with a model in which D-MoDE-A mediates antibody uptake by engaging ASGPR.

In order to determine whether bifunctional small molecule-mediated endocytosis via ASGPR is generalizable to other proteins of interest, we conducted similar experiments with M-MoDE-A and

35 fluorescently labeled human MIF protein (Figure 2D). We observed increased intracellular fluorescence with increasing concentrations of M-MoDE-A, with maximal fluorescence observed at the highest concentration we investigated $(1.0 \mu \mathrm{M})$. In contrast to D-MoDE-A, we did not observe a prozone effect with M-MoDE-A-mediated MIF uptake. These data support that the MoDE-A approach can be generalized to proteins other than $\alpha$-DNP antibody.

To explore the cellular mechanism of antibody uptake, we next treated cells with chemical inhibitors of several endocytic pathways (Figure 2E). Combined treatment with the global endocytosis inhibitors sodium azide and 2-deoxyglucose (DOG) (31) significantly decreased intracellular fluorescence. Fluorescence was also significantly decreased by reagents that inhibit 45 clathrin-dependent endocytosis by disrupting endosomal formation (sucrose (32)) and/or acidification (bafilomycin (33), chloroquine (33), and monensin (34)). In contrast, inhibitors of 
caveolae-mediated endocytosis (nystatin (35)) and macropinocytosis and phagocytosis (cytochalasin D (36), 5-(N-Ethyl-N-isopropyl)amiloride (EIPA), and amiloride (37)) did not significantly decrease cellular fluorescence. The pattern of inhibition observed in these experiments is consistent with an endocytic mechanism that relies on clathrin, but not other endocytic pathways. Given that previous reports have shown that endocytosis mediated by ASGPR is dependent on clathrin $(38,39)$, this data further supports ASGPR's participation in antibody endocytosis mediated by D-MoDE-A. 
A
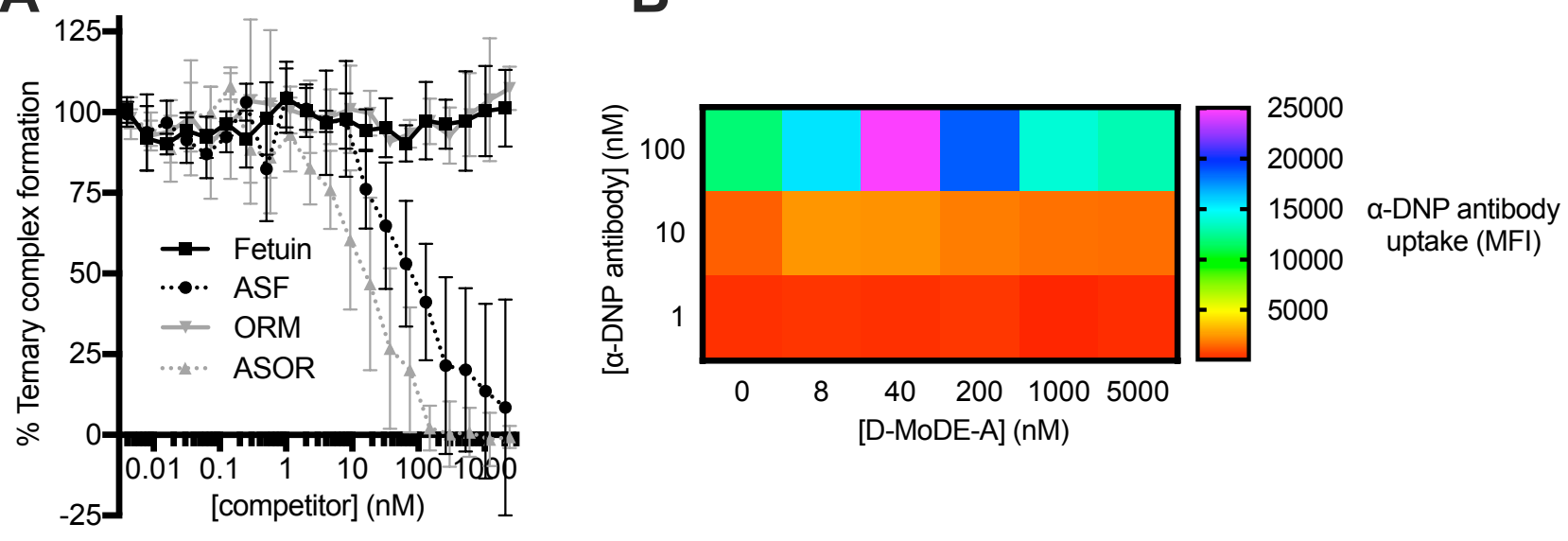

$\mathrm{C}$

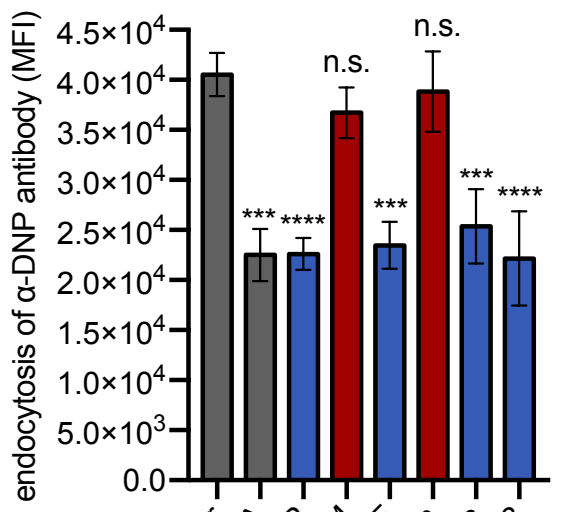

D

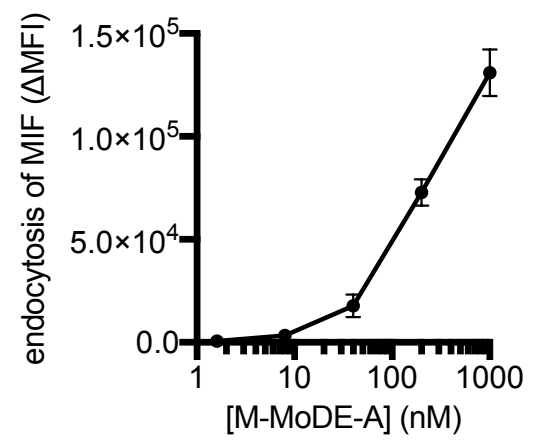

E
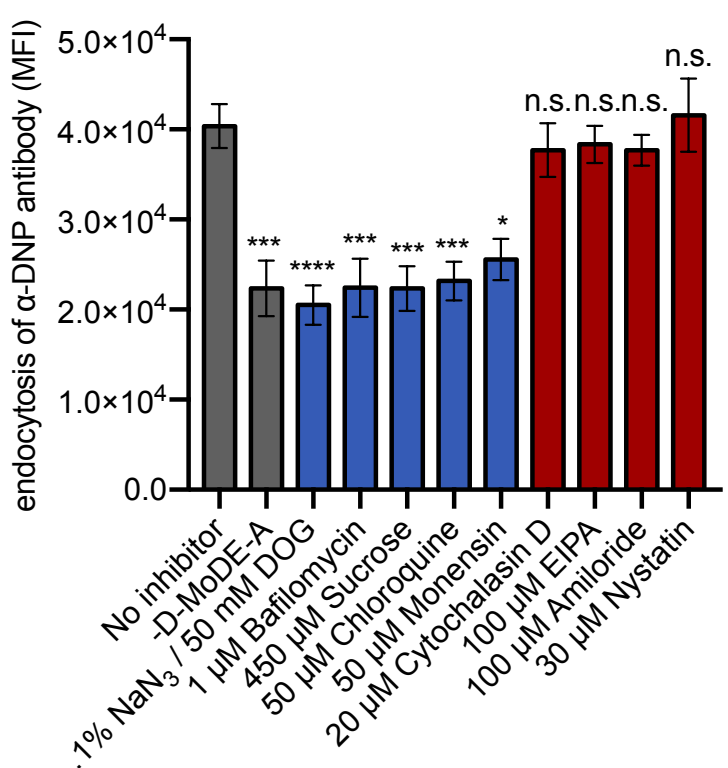
Figure 2: Bifunctional MoDE-A molecules mediate endocytosis of target proteins

A) Ternary complex formation mediated by D-MoDE-A is inhibited by known ASGPR-binding proteins asialofetuin and asialoorosomucoid.

B) Intracellular fluorescence is dependent on the concentrations of both $\alpha$-DNP antibody and D-MoDE-A (6 hour incubation).

C) Endocytosis mediated by D-MoDE-A is decreased by competitive binders of either ASGPR or $\alpha$-DNP antibody. Controls are grey, compounds expected to inhibit the proposed mode of action of D-MoDE-A are blue, and compounds not expected to inhibit are red. Data are presented as mean \pm SD of 9 replicates over four experiments. Statistical differences were determined by Kruskall-Wallace test with post-hoc

10 comparisons between each inhibitor and the no-inhibitor group $(* \mathrm{P}<0.05, * * \mathrm{P}<0.01, * * * \mathrm{P}<0.0001$, $* * * * \mathrm{P}<0.0001$, "n.s"P $>0.9999$ ).

D) Endocytosis of fluorescently labeled MIF is dependent on M-MoDE-A concentration.

E) Inhibitors of clathrin-dependent endocytosis decrease D-MoDE-A-mediated $\alpha$-DNP antibody uptake. Data are presented as mean \pm SD of 9 replicates over four experiments. Statistics were performed as outlined 15 in Figure 2C. 
Having demonstrated that D-MoDE-A mediates the endocytosis of $\alpha$-DNP antibodies, we sought to investigate the subcellular localization of endocytosed fluorescently labeled $\alpha$-DNP antibody. Intracellular antibody-derived fluorescence was found to depend on the presence of both $\mathrm{D}$ MoDE-A and $\alpha$-DNP antibody (Supplemental Figure 3A). We did not observe colocalization of endocytosed antibody with the early endosome marker EEA1 in cells (Figure 3A). In contrast, we observed strong colocalization of antibody with the lysosome membrane protein LAMP2 (Figure 3B). Taken together, these microscopy data suggest that trafficking to lysosomes is rapid on the time scale of this experiment (40) and that the majority of endocytosed antibody was present in mature endocytic compartments.

In addition, we used western blotting with an antibody directed to Alexa Fluor 488 to determine if endocytosed fluorescently labeled $\alpha$-DNP antibodies are degraded in vitro. Lysates from HepG2 cells treated with both D-MoDE-A and $\alpha$-DNP antibody were found to accumulate fluorophore in a time- and D-MoDE-A-dependent manner (Figure 3C). Alexa Fluor 488 in cell lysates was

15 associated with only full-length antibody after two hours of incubation. After six hours, a lower molecular weight fluorophore-associated protein fragment between 37 and $50 \mathrm{kDa}$ began to appear. An additional protein fragment with a molecular weight between 25 and $37 \mathrm{kDa}$ was also observed after 24 hours. We believe that these fluorophore-associated protein fragments are degradation products resulting from lysosomal proteolysis of $\alpha$-DNP antibodies, collectively indicating that endocytosed antibody is degraded in HepG2 cells. Furthermore, no degradation products were observed in the cell supernatant (Supplemental Figure 3B) suggesting that antibody degradation is taking place in or on HepG2 cells. Together with the above immunofluorescence studies showing colocalization of antibody-derived fluorescence with lysosomes, these studies support that antibody degradation is mediated by lysosomal proteases. Lysosomal degradation is further consistent with a mechanism dependent on ASGPR. 


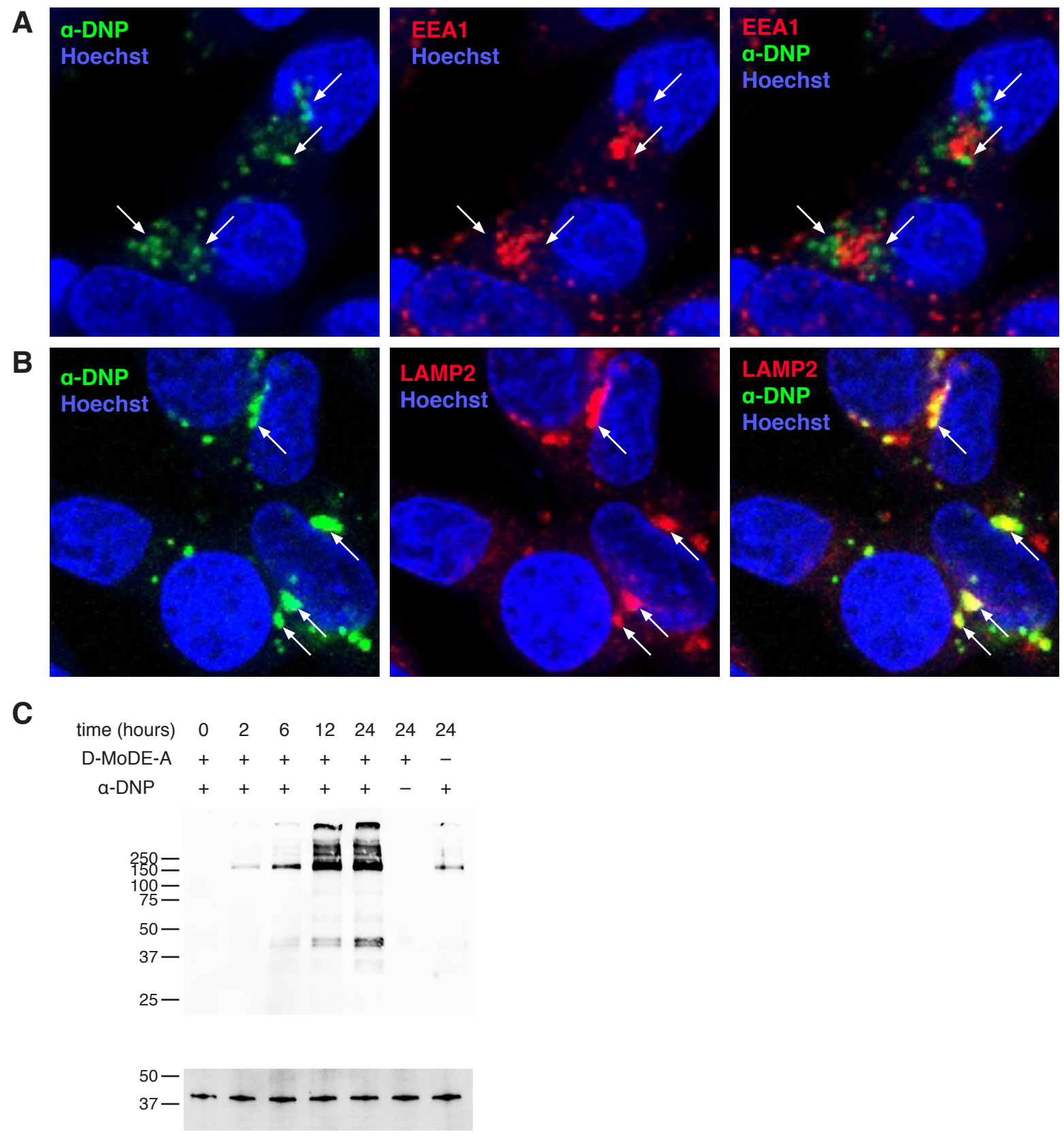

Figure 3: Target protein $\alpha$-DNP antibody is trafficked to lysosomes and degraded

A) Endocytosed $\alpha$-DNP antibody does not colocalize with the early endosome marker EEA1.

B) Endocytosed $\alpha$-DNP antibody colocalizes with the late endosome and lysosome protein LAMP2.

C) Representative western blot showing accumulation of antibody-derived protein fragments in cell lysates. 
Having demonstrated that D-MoDE-A mediates the degradation of $\alpha$-DNP antibodies in vitro, we sought to evaluate the viability of the MoDE-A technology in vivo. A dose of $1 \mathrm{mpk}$ D-MoDE-A was found to be bioavailable via IP dosing in nude mice, with the maximal serum concentration reached after $1 \mathrm{~h}$ and a measured half-life in serum of $0.67 \mathrm{~h}$. D-MoDE-A was well-tolerated up to doses of $100 \mathrm{mpk}$, with no significant differences in body weight or serum liver enzyme levels between control and treatment groups (Supplemental Figure 4A-C). These results supported the suitability of D-MODA-A for more detailed studies in vivo.

Treatment with D-MoDE-A was found to accelerate the depletion of monoclonal mouse IgG2 $\alpha$ DNP antibodies from serum in nude mice in vivo (Figure 4A). Following an initial dose of $200 \mu \mathrm{g}$ $\alpha$-DNP antibody, both daily and twice-daily injections of $1 \mathrm{mpk}$ D-MoDE-A significantly reduced antibody levels compared to PBS treatment over 21 days. Daily treatment with D-MoDE-A also gave a significant decrease in antibody levels following an initial antibody dose of $500 \mu \mathrm{g}$, indicating that D-MoDE-A is effective over a range of target protein concentrations in vivo.

We did not observe accelerated antibody depletion from serum following treatment with the negative control compound DNP-OH3, which binds to $\alpha$-DNP antibody but not ASGPR. Treatment with $10 \mathrm{mpk}$ of DNP-OH3 did not lead to accelerated antibody depletion from serum (Figure 4B), supporting that ASGPR binding is required for target protein depletion in vivo.

20 Unexpectedly, a dose of $100 \mathrm{mpk}$ DNP-OH3 resulted in a small but statistically significantly decrease in antibody clearance compared to the PBS control. We hypothesize that this is a result of an increase in the antibody's hydrodynamic radius due to the DNP-OH3 PEG chains, which may increase its half-life in vivo. Related phenomena have been widely observed for pegylated proteins, and have been exploited for increasing the half lives of various therapeutic modalities 25 (41).

Single doses of D-MoDE-A were also found to be efficacious at mediating $\alpha$-DNP antibody depletion, albeit less effectively than daily dosing (Supplemental Figure 4D). Treatment with either $1 \mathrm{mpk}$ or $10 \mathrm{mpk}$ D-MoDE-A were found to be the most effective, with $52 \%$ and $34 \%$ of $\alpha$ -

30 DNP antibody depleted from serum respectively 24 hours after a single dose, versus $24 \%$ depletion in the vehicle control. Significant depletion was also observed following a dose of $100 \mathrm{mpk}$ of DMoDE-A. We therefore concluded that D-MoDE-A is able to mediate the depletion of a monoclonal antibody from serum, and functions across a wide range of target protein concentrations and dosing regimens.

D-MoDE-A was also found to be efficacious in depleting polyclonal $\alpha$-DNP antibody from serum collected from mice immunized with DNP-KLH (Figure 4C). Following daily treatment with DMoDE-A, significantly more polyclonal $\alpha$-DNP antibody was removed from serum compared to the PBS control at each time point. Thus, the small molecule D-MoDE-A is not restricted in 40 function to only monoclonal mouse antibodies, but is also effective at removing polyclonal $\alpha$-DNP antibodies from circulation in mice. 

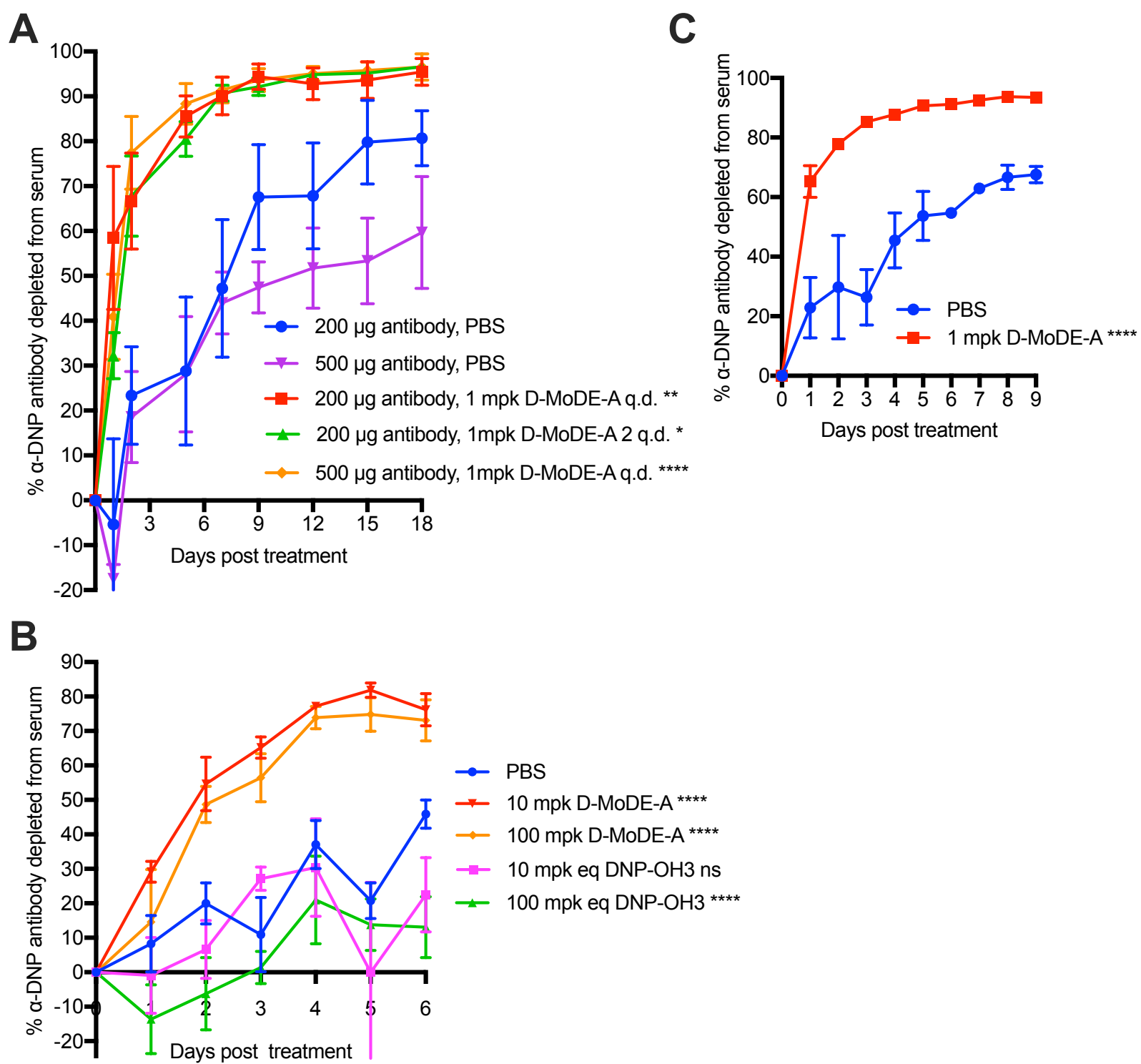

Figure 4: D-MoDE-A mediates the accelerated depletion of $\alpha$-DNP antibodies from serum in vivo

5 A) Serum levels of $\alpha$-DNP antibody as measured by ELISA decrease more rapidly following repeated treatment with D-MoDE-A. Each experimental group contained three mice. Statistical differences in experiments involving in vivo depletion of $\alpha$-DNP antibody were assessed by repeated measures two-way ANOVA with Tukey's tests for post-hoc comparison of simple effects between each of the treatment groups and PBS.

10 B) Significant decreases in serum levels of $\alpha$-DNP antibody are observed after treatment with DMoDE-A, but not DNP-OH3. Each experimental group contained at least five mice.

C) Treatment with D-MoDE-A decreases serum levels of polyclonal $\alpha$-DNP antibody. The PBS treated group contained two mice, while the D-MoDE-A group contained three mice. 
To our knowledge, the MoDE-A technology represents the first demonstration that nonproteinogenic synthetic molecules can effectively mediate the degradation of extracellular proteins. The data presented herein demonstrate that such molecules can induce targeted degradation of structurally diverse proteins, both in tissue culture and in vivo. Recently, Bertozzi and colleagues disclosed the lysosome targeting chimeras (LYTACs) technology, an elegant approach for degrading extracellular and cell surface proteins (42). LYTACs comprise antibodies modified with polymeric ligands of the cation independent mannose-6-phosphate receptor (CIM6PR). Although we believe that the LYTAC approach will likely complement what we present herein, MoDE-A compounds possess several advantages: they are relatively small in size, monodisperse, and non-protein based. These attributes are expected to facilitate synthesis and rapid application to targeting other proteins of interest.

The therapeutic potential of small molecule-based approaches to TPD are particularly evident in light of the recent clinical development of proteolysis targeting chimeras (PROTACs) (43). PROTACs and related technologies induce the formation of ternary complexes between target 15 proteins and E3 ligases such that target proteins are ubiquitinated and subsequently degraded by the proteasome. Due to their mechanism, however, PROTACs have a critical limitation: they cannot degrade targets that are not exposed to the cytosol.

The MoDE-A compounds developed in this work expand the possible targets of small-molecule mediated protein degradation substantially by expanding the scope of TPD to non-cytosolic, extracellular proteins. We believe that MoDE-A compounds will be particularly effective because they direct proteins targets primarily to hepatocytes, a cell type equipped to catabolize large amounts of endocytosed proteins. Furthermore, evidence suggests that ASGPR is tolerogenic immunologically $(44,45)$, which would minimize the likelihood of inducing autoimmune responses to targeted proteins. We expect that the positive pharmacological and biological characteristics of MoDE-A compounds, as well as the straightforward, modular synthetic routes we have developed for their syntheses, will enable rapid optimization and adoption of the MoDEA technology in diverse, disease-relevant contexts. 
Data and materials availability: All data is available in the main text or the supplementary materials.

\section{Supplementary Materials:}

5 Biological Materials and Methods

Synthetic Methods

Figures S1-S4

Spectral Data

Full reference list

10

Acknowledgments: We thank C. Paulsen and S. Miller for use of equipment and the Eshhar Lab for providing hybridomas for antibody production.

Funding: We are thankful for financial support from the National Institute of Health Yale Chemical Biology Training Grant to JDR (2T32GM06754 3-17), EMJB (5T32GM06754 3-12),

15 and DFC (T32GM067543), the Department of Defense (BC120554), and Yale University (Michele Dufault Summer Research Fellowship to AZG).

Competing Interests: This work is the subject of two published patents: PCT/US2019/26239 and PCT/US2019/26260. DAS is a founder of and has equity in Kleo Pharmaceuticals, an entity focused on the development of bifunctional molecules. DAS also receives honoraria for consulting for Kleo. Kleo has licensed intellectual property from DAS, which is used in this research. 


\section{References and Notes:}

1. B. Chen, X. Shi, Y. Cui, A. Hou, P. Zhao, A Review of PCSK9 Inhibitors and their Effects on Cardiovascular Diseases. Curr. Top. Med. Chem. 19, 1790-1817 (2019).

2. G. D. Kalliolias, L. B. Ivashkiv, TNF biology, pathogenic mechanisms and emerging

3. J. B. Bilsborrow, E. Doherty, P. V. Tilstam, R. Bucala, Macrophage migration inhibitory factor (MIF) as a therapeutic target for rheumatoid arthritis and systemic lupus erythematosus. Expert Opin. Ther. 23, 733-744 (2019).

4. J. Martínez-Fábregas, A. Prescott, S. van Kasteren, D. L. Pedrioli, I. McLean, A. Moles, T. Reinheckel, V. Poli, C. Watts, Lysosomal protease deficiency or substrate overload induces an oxidative-stress mediated STAT3-dependent pathway of lysosomal homeostasis. Nat. Commun. 9, 5343 (2018).

5. C. Zoja, A. Benigni, G. Remuzzi, Protein overload activates proximal tubular cells to release vasoactive and inflammatory mediators. Nephron Exp. Nephrol. 7, 420-428 (1999).

6. H. Nakajima, M. Takenaka, J. Y. Kaimori, T. Hamano, H. Iwatani, T. Sugaya, T. Ito, M. Hori, E. Imai, Activation of the signal transducer and activator of transcription signaling pathway in renal proximal tubular cells by albumin. J. Am. Soc. Nephrol. 15, 276-285 (2004).

20 7. M. Tanowitz, L. Hettrick, A. Revenko, G. A. Kinberger, T. P. Prakash, P. P. Seth, Asialoglycoprotein receptor 1 mediates productive uptake of $\mathrm{N}$-acetylgalactosamineconjugated and unconjugated phosphorothioate antisense oligonucleotides into liver hepatocytes. Nucleic Acids Res. 45, 12388-12400 (2017).

8. P. K. Grewal, "The Ashwell-Morell Receptor" in Methods in Enzymology. (Elsevier, 2010), vol. 479, pp. 223-241.

9. Y. Mi, A. Lin, D. Fiete, L. Steirer, J. U. Baenziger, Modulation of mannose and asialoglycoprotein receptor expression determines glycoprotein hormone half-life at critical points in the reproductive cycle. J. Biol. Chem. 289, 12157-12167 (2014).

10. W. H. Yang, P. V. Aziz, D. M. Heithoff, M. J. Mahan, J. W. Smith, J. D. Marth, An intrinsic mechanism of secreted protein aging and turnover. Proc. Natl. Acad. Sci. 112, 13657-13662 (2015).

11. L. M. Steirer, E. I. Park, R. R. Townsend, J. U. Baenziger, The asialoglycoprotein receptor regulates levels of plasma glycoproteins terminating with sialic acid $\alpha 2,6$ galactose. J. Biol. Chem. 284, 3777-3783 (2009).

35 12. R. Grozovsky, A. J. Begonja, K. Liu, G. Visner, J. H. Hartwig, H. Falet, K. M. Hoffmeister, The Ashwell-Morell receptor regulates hepatic thrombopoietin production via JAK2-STAT3 signaling. Nat. Med. 21, 47 (2015).

13. X. Huang, J. C. Leroux, B. Castagner, Well-defined multivalent ligands for hepatocytes targeting via asialoglycoprotein receptor. Bioconjugate Chem. 28, 283-295 (2016).

40 14. A. A. D'Souza, P. V. Devarajan, Asialoglycoprotein receptor mediated hepatocyte targeting-strategies and applications. J. Control. Release 203, 126-139 (2015).

15. S. Matsuda, K. Keiser, J. K. Nair, K. Charisse, R. M. Manoharan, P. Kretschmer, C. G. Peng, A. V. Kel'in, P. Kandasamy, J. L. S. Willoughby, A. Liebow, W. Querbes, K. Yucius, T. Nguyen, S. Milstein, M. A. Maier, K. G. Rajeev, M. Manoharan, siRNA conjugates carrying sequentially assembled trivalent $\mathrm{N}$-acetylgalactosamine linked 
through nucleosides elicit robust gene silencing in vivo in hepatocytes. ACS Chem. Biol. 10, 1181-1187 (2015).

16. E. A. Biessen, A. R. Valentijn, R. L. De Vrueh, E. Van De Bilt, L. A. Sliedregt, P. Prince, M. K. Bijsterbosch, J. H. Van Boom, G. A. Van Der Marel, P. J. Abrahams, T. J. Van Berkel, Novel hepatotrophic prodrugs of the antiviral nucleoside 9-(2phosphonylmethoxyethyl) adenine with improved pharmacokinetics and antiviral activity. FASEB J. 14, 1784-1792 (2000).

17. A. M. Pujol, M. Cuillel, O. Renaudet, C. Lebrun, P. Charbonnier, D. Cassio, C. Gateau, P. Dumy, E. Mintz, P. Delangle, Hepatocyte targeting and intracellular copper chelation by a thiol-containing glycocyclopeptide. J. Am. Chem. Soc. 133, 286-296 (2010).

18. A. Akinc, W. Querbes, S. De, J. Qin, M. Frank-Kamenetsky, K. N. Jayaprakash, M. Jayaraman, K. G. Rajeev, W. L. Cantley, J. R. Dorkin, J. S. Butler, L. Qin, T. Racie, A. Sprague, E. Fava, A. Zeigerer, M. J. Hope, M. Zerial, D. W. Y. Sah, K. Fitzgerald, M. A. Tracy, M. Manoharan, V. Koteliansky, A. de Fuogerolles, M. A. Maier, Targeted delivery of RNAi therapeutics with endogenous and exogenous ligand-based mechanisms. Mol. Ther. 18, 1357-1364 (2010).

19. V. Bocci, The role of sialic acid in determining the life-span of circulating cells and glycoproteins. Experientia 32, 135-140 (1976).

20. J. U. Baenziger, D. Fiete, Galactose and N-acetylgalactosamine-specific endocytosis of

21. J. R. Merwin, G. S. Noell, W. L. Thomas, H. C. Chiou, M. E. DeRome, T. D. McKee, G. L. Spitalny, M. A. Findeis, Targeted delivery of DNA using YEE (GalNAcAH) 3 , a synthetic glycopeptide ligand for the asialoglycoprotein receptor. Bioconjugate Chem. 5, 612-620 (1994).

25 22. P.C. Rensen, L. A. Sliedregt, M. Ferns, E. Kieviet, S. M. Van Rossenberg, S. H. van Leeuwen, T. J. Van Berkel, E. A. Biessen, Determination of the upper size limit for uptake and processing of ligands by the asialoglycoprotein receptor on hepatocytes in vitro and in vivo. J. Biol. Chem. 276, 37577-37584 (2001).

23. S. K. Mamidyala, S. Dutta, B. A. Chrunyk, C. Préville, H. Wang, J. M. Withka, A. McColl, T. A. Subashi, S. J. Hawrylik, M. C. Griffor, S. Kim, J. A. Pfefferkorn, D. A. Price, E. Menhaji-Klotz, V. Mascitti, M. G. Finn, Glycomimetic ligands for the human asialoglycoprotein receptor. J. Am. Chem. Soc. 134, 1978-1981 (2012).

24. T. Onizuka, H. Shimizu, Y. Moriwaki, T. Nakano, S. Kanai, I. Shimada, H. Takahashi, NMR study of ligand release from asialoglycoprotein receptor under solution conditions in early endosomes. FEBS J. 279, 2645-2656 (2012).

25. J. H. LaBadie, K. P. Chapman, N. N. Aronson, Glycoprotein catabolism in rat liver: Lysosomal digestion of iodinated asialo-fetuin. Biochem. J. 152, 271-279 (1975).

26. J. A. Cisneros, M. J. Robertson, M. Valhondo, W. L. Jorgensen, A fluorescence polarization assay for binding to macrophage migration inhibitory factor and crystal structures for complexes of two potent inhibitors. J. Am. Chem. Soc. 138, 8630-8638 (2016).

27. E. F. Douglass Jr, C. J. Miller, G. Sparer, H. Shapiro, D. A. Spiegel, A comprehensive mathematical model for three-body binding equilibria. J. Am. Chem. Soc. 135, 6092-6099 (2013).

45 28. W. Brown, N. Saunders, K. Møsllgård, K. Dziegielewska, Fetuin-an old friend revisited. BioEssays 14, 749-755 (1992). 
29. C. L. Fernandes, R. Ligabue-Braun, H. Verli, Structural glycobiology of human $\alpha_{1}$-acid glycoprotein and its implications for pharmacokinetics and inflammation. Glycobiology 25, 1125-1133 (2015).

30. B. Shi, M. Abrams, L. Sepp-Lorenzino, Expression of Asialoglycoprotein Receptor 1 in

31. A. Cooper, Y. Shaul, Clathrin-mediated endocytosis and lysosomal cleavage of hepatitis B virus capsid-like core particles. J. Biol. Chem. 281, 16563-16569 (2006).

32. S. Guo, X. Zhang, M. Zheng, X. Zhang, C. Min, Z. Wang, S. H. Cheon, M. H. Oak, S. Y. Nah, K. M. Kim, Selectivity of commonly used inhibitors of clathrin-mediated and caveolae-dependent endocytosis of $\mathrm{G}$ protein-coupled receptors. BBA-Biomembranes 1848, 2101-2110 (2015).

33. L. D. Cervia, C. C. Chang, L. Wang, F. Yuan, Distinct effects of endosomal escape and inhibition of endosomal trafficking on gene delivery via electrotransfection. PloS One 12 (2017).

15 34. R. Ippoliti, P. Ginobbi, E. Lendaro, I. D’Agostino, D. Ombres, P. A. Benedetti, M. Brunori, G. Citro, The effect of monensin and chloroquine on the endocytosis and toxicity of chimeric toxins. Cell. Mol. Life Sci. 54, 866-875 (1998).

35. Q. Ba, N. Zhou, J. Duan, T. Chen, M. Hao, X. Yang, J. Li, J. Yin, R. Chu, H. Wang, Dihydroartemisinin exerts its anticancer activity through depleting cellular iron via transferrin receptor-1. PloS One 7 (2012).

36. M. M. Zegers, K. J. Zaal, S. C. van IJzendoorn, K. Klappe, D. Hoekstra, Actin filaments and microtubules are involved in different membrane traffic pathways that transport sphingolipids to the apical surface of polarized HepG2 cells. Mol. Biol. Cell 9, 1939-1949 (1998).

25 37. R. Maidorn, E. Cragoe, I. Tannock, Therapeutic potential of analogues of amiloride: inhibition of the regulation of intracellular $\mathrm{pH}$ as a possible mechanism of tumour selective therapy. Br. J. Cancer 67, 297-303 (1993).

38. J. Oka, M. Christensen, P. Weigel, Hyperosmolarity inhibits galactosyl receptor-mediated but not fluid phase endocytosis in isolated rat hepatocytes. J. Biol. Chem. 264, 1201612024 (1989).

39. A. L. Schwartz, A. Bolognesi, S. E. Fridovich, Recycling of the asialoglycoprotein receptor and the effect of lysosomotropic amines in hepatoma cells. J. Cell Biol. 98, 732738 (1984).

40. K. Bridges, J. Harford, G. Ashwell, R. D. Klausner, Fate of receptor and ligand during endocytosis of asialoglycoproteins by isolated hepatocytes. Proc. Natl. Acad. Sci. 79, 350-354 (1982).

41. S. Jevsevar, M. Kunstelj, "Half-life extension through PEGylation" in Therapeutic Proteins: Strategies to Modulate Their Plasma Half-lives, R. Kontermann, Ed. (WileyBlackwell, 2012), chap. 3.

40 42. S. Banik, K. Pedram, S. Wisnovsky, N. Riley, C. Bertozzi, https://chemrxiv.org/articles/preprint/Lysosome_Targeting_Chimeras_LYTACs_for_the_ Degradation_of_Secreted_and_Membrane_Proteins/7927061/2 (2019).

43. X. Sun, H. Gao, Y. Yang, M. He, Y. Wu, Y. Song, Y. Tong, Y. Rao, PROTACs: great opportunities for academia and industry. Sig. Transduct. Target Ther. 4, 64 (2019).

45 44. C. S. Guy, S. L. Rankin, T. I. Michalak, Hepatocyte cytotoxicity is facilitated by asialoglycoprotein receptor. Hepatol. 54, 1043-1050 (2011). 
45. V. Benseler, A. Warren, M. Vo, L. E. Holz, S. S. Tay, D. G. Le Couteur, E. Breen, A. C. Allison, N. van Rooijen, C. McGuffog, H. J. Schlitt, D. G. Bowen, G. W. McCaughan, P. Bertolino, Hepatocyte entry leads to degradation of autoreactive CD8 T cells. Proc. Natl. Acad. Sci. 108, 16735-16740 (2011).

5 46. P. Weigel, J. Oka, Temperature dependence of endocytosis mediated by the asialoglycoprotein receptor in isolated rat hepatocytes. Evidence for two potentially ratelimiting steps. J. Biol. Chem. 256, 2615-2617 (1981).

47. Z. Eshhar, M. Ofarim, T. Waks, Generation of hybridomas secreting murine reaginic antibodies of anti-DNP specificity. J. Immunol. Res. 124, 775-780 (1980).

10 48. K. Heller, P. Ochtrop, M. F. Albers, F. B. Zauner, A. Itzen, C. Hedberg, Covalent protein labeling by enzymatic phosphocholination. Angew. Chem. Int. Ed. Engl. 54, 10327-10330 (2015).

49. J. Iglesias-Fernández, S. M. Hancock, S. S. Lee, M. Khan, J. Kirkpatrick, N. J. Oldham, K. McAuley, A. Fordham-Skelton, C. Rovira, B. G. Davis, A front-face 'S $\mathrm{S}_{\mathrm{N}}$ synthase'

15 engineered from a retaining 'double-S $\mathrm{N}_{\mathrm{N}}$ ' hydrolase. Nat. Chem. Bio. 13, 874 (2017).

50. P. C. Rensen, S. H. van Leeuwen, L. A. Sliedregt, T. J. van Berkel, E. A. Biessen, Design and synthesis of novel $\mathrm{N}$-acetylgalactosamine-terminated glycolipids for targeting of lipoproteins to the hepatic asialoglycoprotein receptor. J. Med. Chem. 47, 5798-5808 (2004).

20 51. N. Avlonitis, M. Debunne, T. Aslam, N. McDonald, C. Haslett, K. Dhaliwal, M. Bradley, Highly specific, multi-branched fluorescent reporters for analysis of human neutrophil elastase. Org. Biomol. Chem. 11, 4414-4418 (2013).

52. G. J. Miller, J. M. Gardiner, Adaptable synthesis of C-glycosidic multivalent carbohydrates and succinamide-linked derivatization. Org. Lett. 12, 5262-5265 (2010).

25 53. J. A. Cisneros, M. J. Robertson, M. Valhondo, W. L. Jorgensen, A fluorescence polarization assay for binding to macrophage migration inhibitory factor and crystal structures for complexes of two potent inhibitors. J. Am. Chem. Soc. 138, 8630-8638 (2016).

54. C. A. DeForest, D. A. Tirrell, A photoreversible protein-patterning approach for guiding stem cell fate in three-dimensional gels. Nat. Mater. 14, 523-531 (2015).

55. L. Liao, J. Liu, E. C. Dreaden, S. W. Morton, K. E. Shopsowitz, P. T. Hammond, J. A. Johnson, A convergent synthetic platform for single-nanoparticle combination cancer therapy: ratiometric loading and controlled release of cisplatin, doxorubicin, and camptothecin. J. Am. Chem. Soc. 136, 5896-5899 (2014).

35 56. D. M. Tian, J. Qiao, Y. Z. Bao, J. Liu, X. K. Zhang, X. L. Sun, Y. W. Zhang, X. S. Yao, J. S. Tang, Design and synthesis of biotinylated cardiac glycosides for probing Nur77 protein inducting pathway. Bioorg. Med. Chem. Lett. 29, 707-712 (2019). 